\section{AB0719 ASSOCIATION BETWEEN FIBROMYALGIA AND CHRONIC AUTOIMMUNE THYROIDITIS. RETROSPECTIVE OBSERVATIONAL DATA FROM A MONOCENTRIC ENDOCRINOLOGIST- RHEUMATOLOGIST COLLABORATIVE ANALYSIS}

F. Fischetti ${ }^{1}$, S. Bernardi2, S. Lovriha2, F. Bozzao2, P. Tomietto ${ }^{3}$, B. Fabris2. ${ }^{1}$ University of Trieste, Department of Medical, Surgery and Health Sciences, Trieste, Italy; ${ }^{1}$ University of Trieste, Department of Medical, Surgery and Health Sciences, Trieste, Italy; ${ }^{3}$ Azienda Sanitaria Universitaria Giuliano Isontina; Cattinara Hospital, Department of Medicine, Trieste, Italy

Background: Endocrine and metabolic imbalance conditions can affect the development of subjective abnormal perceptions within fibromyalgia (FMR). In the case of autoimmune thyroid disease (ATD), prolonged, clinically active states of impaired glandular function may be associated with an FMR-type condition. Less clear is the association between subclinical or rapidly well-controlled states of thyroid disease and the presence of FMR, since this assessment, although analysed in some previous studies, was usually performed on cohorts of subjects where the absence of any other confusing factors was not well defined.

Objectives: To evaluate the prevalence of subclinical autoimmune thyroid disease, or functionally controlled autoimmune thyroid disease, in a retrospective cohort of consecutively diagnosed patients suffering from fibromyalgia condition. Methods: Over a 2 years period of time (2018-2019) a monocentric joint evaluation was activated with the endocrinology section of our healthcare area in order to consecutively monitor the subjects belonging to both specialist clinics. Patients with ATD were not unfrequently firstly evaluated in the rheumatology ambulatory. Diagnosis of FMR was defined according to the American College of Rheumatology 2010/2011 criteria. At the same time, at the rheumatology clinics, all cases addressed with diagnosis of fatigue or chronic pain of no defined origin were analysed in order to carefully identify any associated, comorbidity problems. The diagnosis of ATD was confirmed according to recognized international criteria. The following results will focus on subjects with chronic Hashimoto type Thyroiditis (HT).

Results: Among the HT patients, 98\% were women, aged between 28 and 64 . Over the 2 years considered period of time, 65 subjects suffering from HT, showing no active disease or unstable endocrine function were addressed to the rheumatology clinics owing to FMR related symptoms. Among them, $55(84.6 \%)$ had a confirmed diagnosis of FMR. Within this time, we recognized 239 consecutive diagnoses of FMR in subjects aging 22-76 years, with a number of 114 found to be devoid of factors (other than ATD) able to be responsible for chronic pain, except for a modest component of situational anxiety, or mild mood depression, not requiring any specific drug intervention. Among the 114, so called "primary" FMR, $35.6 \%$ showed to suffer from TCH, under confirmed clinical/hormonal remission, or in a preclinical, early stage of onset. Within the 125 subjects, carrying a FMR condition related to previous or associated fostering pathology, $26.8 \%$ were positive for current or previous thyroid problems. The prevalence of $\mathrm{TCH}$, in the "secondary" FMR conditions differed significantly $(p<0.01)$ from that of other FMR promoting diseases (eg connective tissue diseases, such as Sjogren Syndrome), except for moderate-severe mood disorders and/or anxiety, and the most severe chronic osteoarthritis conditions, showing a confirmed secondary neuropathy.

Conclusion: Although limited in number, the here reported data confirm the hypothesis of a significant association between ATD and FMR, even in subjects who were considered to be in a subclinical condition or in full clinical remission by the endocrinology colleagues. The physiopathology of this association needs further appropriate insights.

Acknowledgements: We thank Dr Alberto Petterle for his previous helpful contribution

Disclosure of Interests: None declared

DOI: 10.1136/annrheumdis-2021-eular.3985

\section{Paediatric rheumatology}

\section{AB0720 HIP INVOLVEMENT IN A COHORT OF EGYPTIAN JUVENILE IDIOPATHIC ARTHRITIS PATIENTS}

A. Radwan ${ }^{1}$, H. Alzahrani ${ }^{2}$, Y. Alzahrani ${ }^{2}$, A. Elsaman ${ }^{3} .{ }^{1}$ Sohag University Hospital, Rheumatology and Rehabilitation, Sohag,; ${ }^{2}$ Armed Forces
Specialized Center for Health Rehabilitation, Altaif, Kingdom of Saudi Arabia, Rehabilitation Depatment, Taif, Saudi Arabia; ${ }^{3}$ Sohag University Hospital, Rheumatology and Rehabilitation, Sohag, Egypt

Background: The hip joint is frequently involved in Juvenile Idiopathic Arthritis (JIA). It is more common with polyarticular, systemic and enthesitis-related forms and with severe uncontrolled disease. Chronic hip arthritis leads to irreversible joint damage with marked impairment of quality of life and functional limitation [1]. Unilateral or bilateral hip arthritis occurs in $30-50 \%$ of children with JIA [2]. Objectives: The aim of this study is to assess the pattern of hip involvement in a cohort of Egyptian JIA patients in terms of epidemiological aspects, JIA pattern, bilateralism, associated extra-articular manifestations, radiological features, treatment and prognosis.

Methods: We included 179 patients who fulfilled the International league against rheumatism criteria for JIA. Epidemiological, clinical, radiological, and therapeutic parameters were assembled and analyzed. Hip involvement was assessed using a semi-quantitative score of pain and tenderness for the hip, CARSH radiographic score of the hip, and Harris functional hip score. JADAS-27 was used for assessment of disease activity.

Results: We included 113 girls and 66 boys; with a female: male ratio of nearly $2: 1$. The age at onset mean was $8.8 \pm 3$ years $(8.9 \pm 2.9$ for females and $8.6 \pm 3.1$ for males). The mean age at the study time was $13.3 \pm 4.1$ years. The disease duration mean was $4.5 \pm 2.9$ years.

Clinically; $20.1 \%$ of the cases had hip involvement (12.8\% unilateral and $7.3 \%$ bilateral), while by imaging, around $30.7 \%$ of the cases have hip involvement ( $19.6 \%$ unilateral and $11.2 \%$ bilateral). The mean age for cases with hip involvement was $14.1 \pm 4.3$, compared to $12.9 \pm 4$ among those with no hip involvement. The mean disease duration for those with hip arthritis (either clinical or by imaging) was $5.5 \pm 2.9$ years, compared to $4.1 \pm 2.9$ among those with no hip involvement (Figure 1).

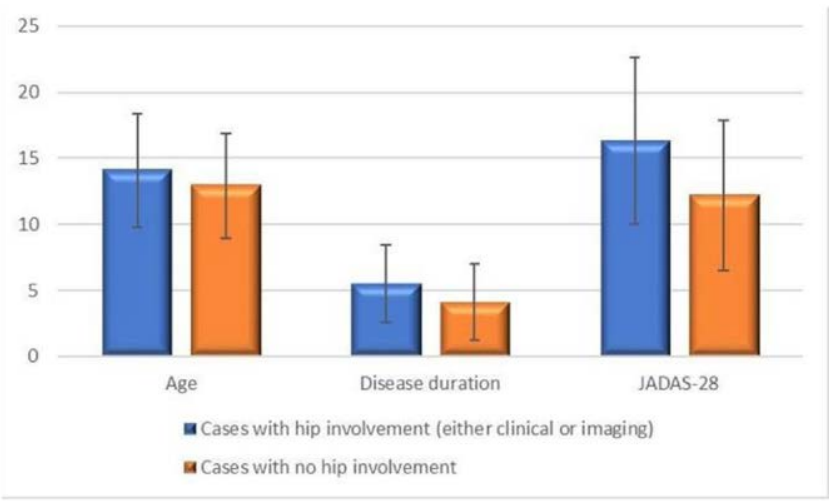

Figure 1. Difference between JIA cases with and without hip involvement.

The Mean JADAS-27 was $13.5 \pm 6.2$ and for those with hip involvement was 16.3 \pm 6.3 . The commonest pattern of JIA with hip arthritis was polyarticular followed by enthesitis-related arthritis. There was a strong significant correlation between JADAS-27 and hip involvement at one hand and Harris score, semi-quantitative score for pain and tenderness at the other hand. Further, disease duration was significantly correlated with hip involvement as well. Among cases with hip involvement, $25 \%$ demonstrated destructive changes and $30 \%$ showed growth abnormalities.

Conclusion: Hip arthritis in JIA is related to polyarticular and enthesitis-related pattern. Longer disease duration, seropositive polyarticular pattern were related to poor prognosis for hip arthritis.

\section{REFERENCES:}

[1] Singh JA, Cleveland JD. Juvenile idiopathic arthritis is associated with higher healthcare utilization after total knee or hip replacement. Scandinavian journal of rheumatology 2021; 50: 34-9.

[2] Rostom S, Amine B, Bensabbah R, Abouqal R, Hajjaj-Hassouni N. Hip involvement in juvenile idiopathic arthritis. Clinical rheumatology 2008; 27: 791-4.

Disclosure of Interests: None declared

DOI: 10.1136/annrheumdis-2021-eular.712 